Supporting information for:

\title{
Monte Carlo simulation of water adsorption in hydrophobic MFI zeolites with hydrophilic sites
}

\author{
M. Göktuğ Ahunbay
}

Department of Chemical Engineering, Istanbul Technical University, 34469 Istanbul, Turkey

Appendix A: Water and Zeolite Potential models

Appendix B: Silicalite structures containing silanol nests 


\section{Appendix A: Water and Water-Zeolite Potential models}

A.1) Water and water-zeolite interaction models proposed by Demontis, Suffritti and and co-workers.

- Water model [1]:

$V_{\mathrm{OO}}=\frac{1}{4 \pi \epsilon_{0}} \frac{q_{\mathrm{O}} q_{\mathrm{o}}}{r}+\frac{A}{r^{12}}-\frac{B}{r^{6}}+\frac{C}{r^{4}}-D \exp \left[-E r-F^{2}\right]$

$V_{\mathrm{OH}}=\frac{1}{4 \pi \epsilon_{0}} \frac{q_{\mathrm{O}} q_{\mathrm{H}}}{r}+A \exp (-B r)-\frac{C}{r^{4}}-D \exp \left[-E r-F^{2}\right]$

$V_{\mathrm{HH}}=\frac{1}{4 \pi \epsilon_{0}} \frac{q_{H} q_{\mathrm{H}}}{r}+A \exp (-B r)-C \exp \left[-D r-E^{2}\right]$

TABLE A.1. Parameters for the water model [Eqs. (1)-(3)].

\begin{tabular}{lcccccc}
\hline Parameters & A & B & C & D & E & F \\
$\mathrm{V}_{\mathrm{OO}}(\mathrm{kJ} / \mathrm{mol})$ & 3500000 & 3100 & 15.0 & 2.093 & 1.5 & 4.5 \\
$\mathrm{~V}_{\mathrm{OH}}(\mathrm{kJ} / \mathrm{mol})$ & 19098.33 & 4.06 & 223.796 & & & \\
$\mathrm{~V}_{\mathrm{HH}}(\mathrm{kJ} / \mathrm{mol})$ & 2134.86 & 2.76 & 4.186 & 1.5 & 3.15 & \\
& & & & & & \\
Charges & & & & & & \\
$\mathrm{q}_{\mathrm{O}}(\mathrm{e})$ & -0.65966 & & & & & \\
$\mathrm{q}_{\mathrm{H}}(\mathrm{e})$ & 0.32983 & & & &
\end{tabular}

- Water-zeolite interaction model [2]:

$V_{\mathrm{OO}_{\mathrm{Z}}}=\frac{1}{4 \pi \epsilon_{0}} \frac{q_{\mathrm{o}} q_{\mathrm{O}_{\mathrm{Z}}}}{r}+\frac{A}{r^{12}}-\frac{B}{r^{6}}+\frac{C}{r^{4}}$

$V_{\mathrm{HO}_{\mathrm{Z}}}=\frac{1}{4 \pi \epsilon_{0}} \frac{q_{\mathrm{H}} q_{\mathrm{O}_{\mathrm{Z}}}}{r}+\frac{A}{r^{7}}-\frac{C}{r^{4}}$

TABLE A.2. Parameters for the water-zeolite interaction model [Eqs. (4)-(5)].

\begin{tabular}{lccc}
\hline Parameters & A & B & C \\
$\mathrm{V}_{\mathrm{OOz}}(\mathrm{kJ} / \mathrm{mol})$ & $8.36 \times 10^{5}$ & 3100 & 209 \\
$\mathrm{~V}_{\mathrm{HOz}}(\mathrm{kJ} / \mathrm{mol})$ & 2090 & 179.9 &
\end{tabular}

\section{Charges}

\begin{tabular}{lc}
$\mathrm{q}_{\mathrm{O}}(\mathrm{e})$ & -1 \\
$\mathrm{q}_{\mathrm{Si}}(\mathrm{e})$ & 2 \\
\hline
\end{tabular}




\section{A.2) Water model propsed by Errington and Panagiotopoulos [3]}

$V=\frac{\epsilon}{1-\frac{6}{\alpha}} \frac{6}{\alpha} \exp \alpha \frac{1-r}{r_{m}}-{\frac{r_{m}}{r}}^{6}$

where $r_{m}$ is the value of $r$ at which $V(r)=0, \varepsilon$ is the well depth (energy), $\alpha$ is the "steepness" of the repulsive energy. This expression can be rewritten in the form of familiar Buckingham potential:

$V=A \exp -B r-\frac{C}{r^{6}}$

with

$A=\frac{6 \epsilon}{\alpha-6} \exp (\alpha)$

$B=\frac{\alpha}{r_{m}}$

$C=\frac{\alpha \epsilon}{\alpha-6} r_{m}^{6}$

\section{A.3) PCFF potential parameters for intra-framework interactions in silicalite [4]}

(Distances in $\AA$, and energies in $\mathrm{kcal} / \mathrm{mol}$ )

Van der Waals:

$V_{V W}=\varepsilon 2{\frac{r_{m}}{r}}^{9}-3{\frac{r_{m}}{r}}^{6}$

$O_{Z}-O_{Z}: \quad r_{m}=3.4506, \varepsilon=0.16220$

\section{Bond Stretch:}

$V_{\text {Bond }}=k_{2} \mathrm{~b}-\mathrm{b}_{0}^{2}+k_{3} \mathrm{~b}-\mathrm{b}_{0}^{3}+k_{4} \mathrm{~b}-\mathrm{b}_{0}^{4}$

$\mathrm{O}_{\mathrm{Z}}-\mathrm{Si}_{\mathrm{Z}}: \mathrm{k}_{2}=325.443, \mathrm{k}_{3}=-943.3640, \mathrm{k}_{4}=1454.67, \mathrm{~b}_{0}=1.6155$. 


\section{Angle Bending:}

$V_{\text {Bend }}=k_{2} \theta-\theta_{0}^{2}+k_{3} \theta-\theta_{0}^{3}+k_{4} \theta-\theta_{0}^{4}$

\begin{tabular}{ccccc} 
& $\theta_{0}$ & $\mathrm{k}_{2}$ & $\mathrm{k}_{3}$ & $\mathrm{k}_{4}$ \\
\cline { 2 - 5 } $\mathrm{O}_{\mathrm{Z}}-\mathrm{Si}_{\mathrm{Z}}-\mathrm{O}_{\mathrm{Z}}:$ & 176.2650 & 18.8146 & 37.9749 & 42.8222
\end{tabular}

$\mathrm{Si}_{Z^{-}} \mathrm{O}_{Z^{-}} \mathrm{Si}_{\mathrm{Z}}: \quad 110.6120 \quad 154.1860 \quad-68.6595 \quad 23.6292$

\section{Torsion}

$V_{\text {Torsion }}=k_{1} 1+\cos \left(\varphi-\varphi_{0}\right)+k_{2} 1+\cos \left(2 \varphi-\varphi_{0}\right)+k_{3} 1+\cos \left(3 \varphi-\varphi_{0}\right)$

$\mathrm{Si}_{\mathrm{Z}}-\mathrm{O}_{\mathrm{Z}}-\mathrm{Si}_{\mathrm{Z}}-\mathrm{O}_{\mathrm{Z}}: \mathrm{k}_{2}=-0.3417, \mathrm{k}_{3}=0.0961, \mathrm{k}_{4}=0.1683, \varphi_{0}=0.0$.

\section{Bond-Bond}

$V_{\text {Bond-bond }}=k \mathrm{~b}-\mathrm{b}_{0} \mathrm{~b}^{\prime}-b_{0}^{\prime}$

$k=178.8840$

Bond-Angle

$V_{\text {Bond-Angle }}=k \mathrm{~b}-\mathrm{b}_{0} \quad \theta-\theta_{0}$

$\mathrm{Si}_{\mathrm{Z}^{-}} \mathrm{O}_{\mathrm{Z}^{-}} \mathrm{Si}_{\mathrm{Z}}: k=13.4905$

$\mathrm{O}_{\mathrm{Z}}-\mathrm{Si}_{\mathrm{Z}}-\mathrm{O}_{\mathrm{Z}}: k=87.3528$

\section{Angle-Angle}

$V_{\text {Angle }}=k \theta-\theta_{0} \quad \theta^{\prime}-\theta_{0}^{\prime}$

$\mathrm{Si}_{\mathrm{Z}}-\mathrm{O}_{\mathrm{Z}}-\mathrm{Si}_{\mathrm{Z}}-\mathrm{O}_{\mathrm{Z}}: k=5.7889$ 

Appendix B: Silicalite structures containing silanol nests

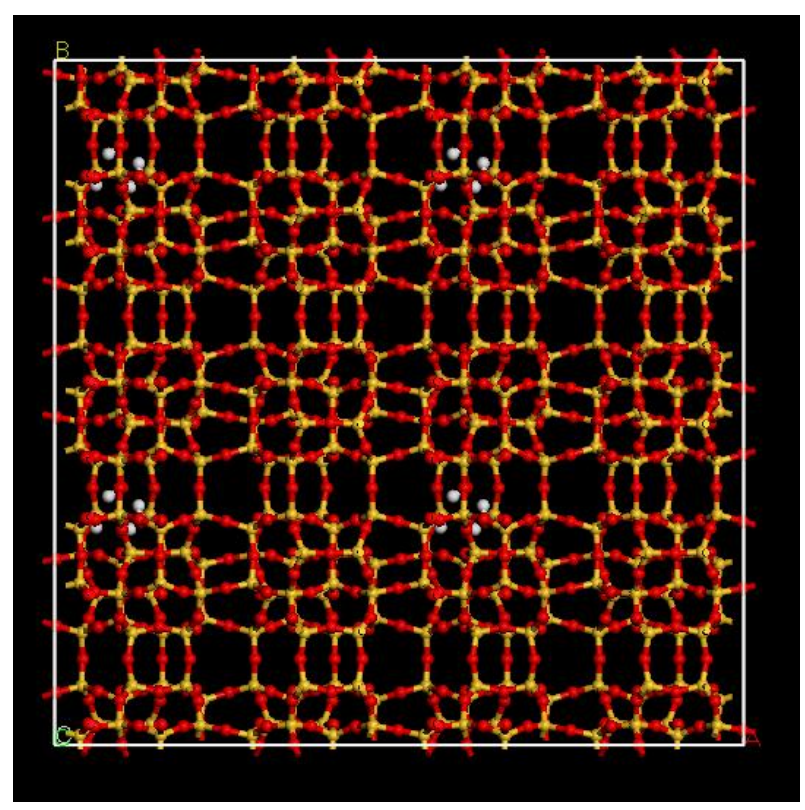

(a)

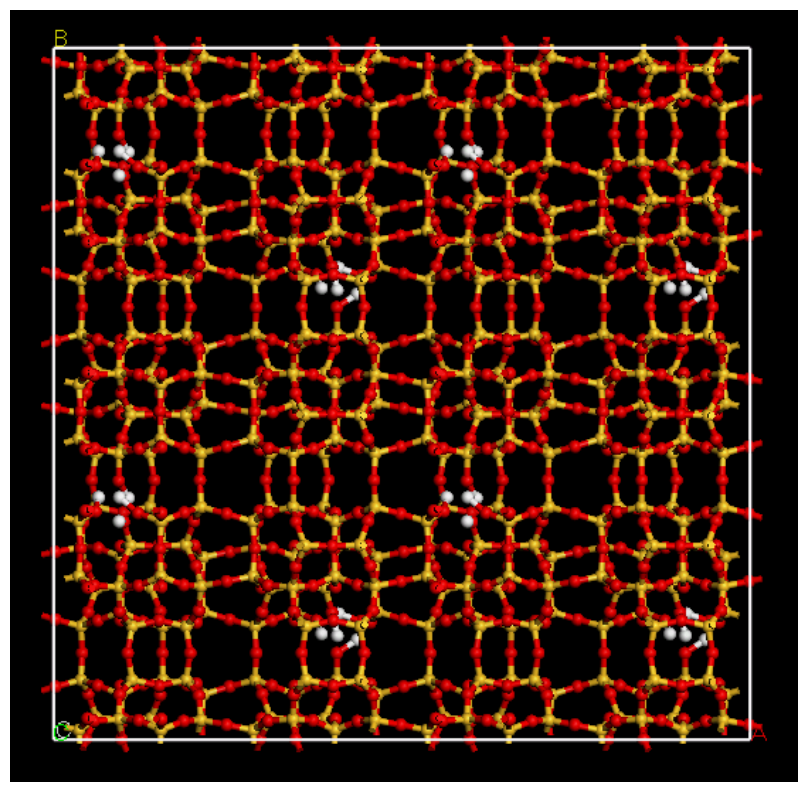

(c)

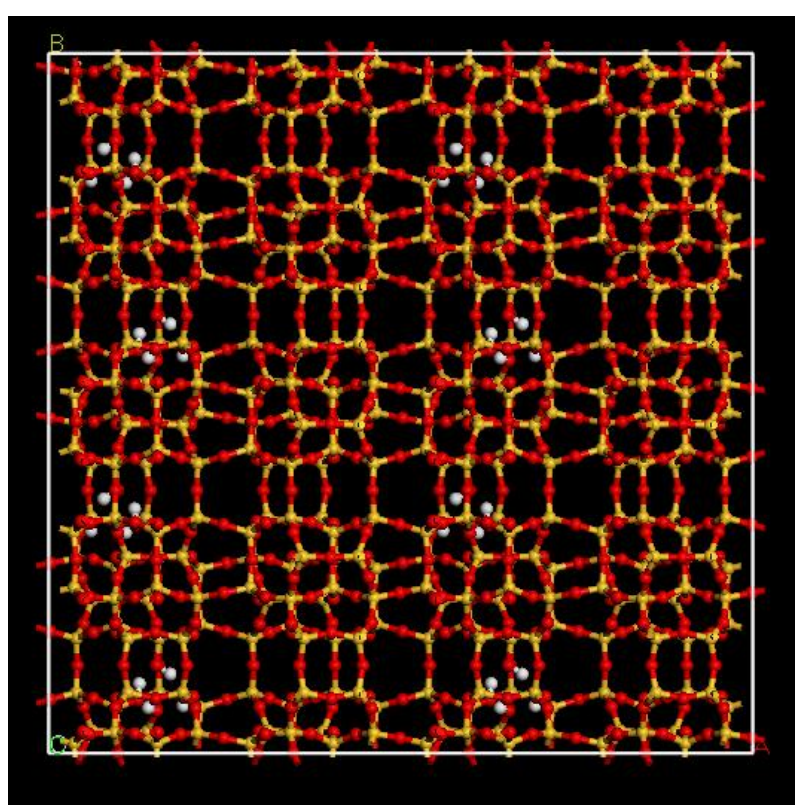

(b)

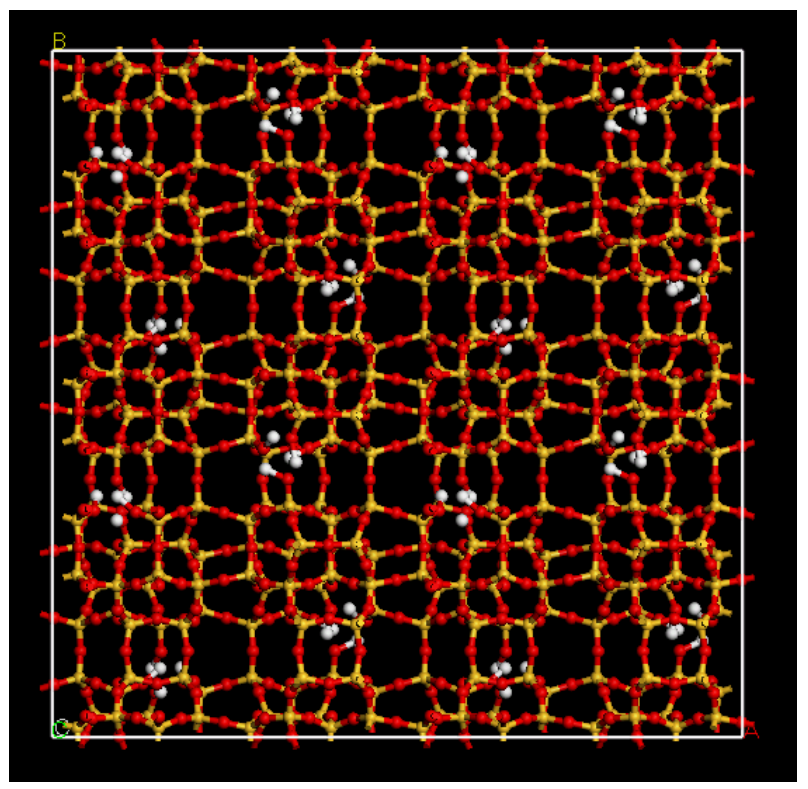

(d)

Figure B1. Silicalite structures containing (a) 0.5, (b) 1, (c) 2, (d) 4 silanol nests per unit cell. 


\section{References:}

1. Cui P., Demontis P., Spanu S., Suffritti G.B., Tilocca A,. J. Phys. Chem. B. 2003, 107, 44264436.

2. Demontis P., Stara G., and Suffritti G.B., J. Phys. Chem. B. 2003, 107, 4426-4436.

3. Errington J.R., Panagiotopoulos A.Z., J. Phys. Chem. B. 1998, 102, 7470.

4. Hill, J.-R.; Sauer, J.; J. Phys. Chem. 1994, 98, 1238. 